$5-1-2012$

\title{
Occupational therapy and sensory integration for children with autism: a feasibility, safety, acceptability and fidelity study.
}

\author{
Roseann C Schaaf \\ Thomas Jefferson University \\ Teal W. Benevides \\ Thomas Jefferson University \\ Donna Kelly \\ Children's Specialized Hospital, Tom's River, New Jersey, USA \\ Zoe Mailloux-Maggio \\ Pediatric Therapy Network, Torrance, California
}

Follow this and additional works at: https://jdc.jefferson.edu/otfp

Part of the Occupational Therapy Commons

Let us know how access to this document benefits you

\section{Recommended Citation}

Schaaf, Roseann C; Benevides, Teal W.; Kelly, Donna; and Mailloux-Maggio, Zoe, "Occupational therapy and sensory integration for children with autism: a feasibility, safety, acceptability and fidelity study." (2012). Department of Occupational Therapy Faculty Papers. Paper 13. https://jdc.jefferson.edu/otfp/13

This Article is brought to you for free and open access by the Jefferson Digital Commons. The Jefferson Digital Commons is a service of Thomas Jefferson University's Center for Teaching and Learning (CTL). The Commons is a showcase for Jefferson books and journals, peer-reviewed scholarly publications, unique historical collections from the University archives, and teaching tools. The Jefferson Digital Commons allows researchers and interested readers anywhere in the world to learn about and keep up to date with Jefferson scholarship. This article has been accepted for inclusion in Department of Occupational Therapy Faculty Papers by an authorized administrator of the Jefferson Digital Commons. For more information, please contact: JeffersonDigitalCommons@jefferson.edu. 


\section{Occupational therapy and sensory integration for children with autism: a feasibility, safety, acceptability and fidelity study}

Roseann C. Schaaf, Teal W. Benevides, Donna Kelly and Zoe Mailloux-Maggio

Autism 2012 16: 321 originally published online 8 February 2012

DOI: $10.1177 / 1362361311435157$

The online version of this article can be found at:

http://aut.sagepub.com/content/16/3/321

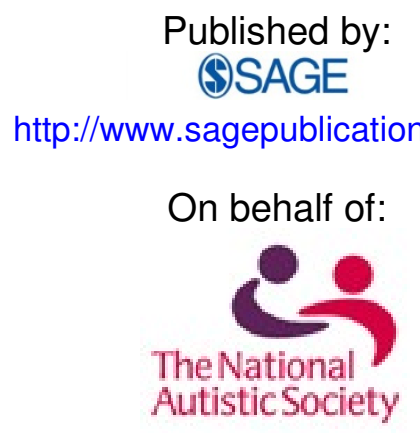

The National Autistic Society

Additional services and information for Autism can be found at:

Email Alerts: http://aut.sagepub.com/cgi/alerts

Subscriptions: http://aut.sagepub.com/subscriptions

Reprints: http://www.sagepub.com/journalsReprints.nav

Permissions: http://www.sagepub.com/journalsPermissions.nav

Citations: http://aut.sagepub.com/content/16/3/321.refs.html

>> Version of Record - May 24, 2012

OnlineFirst Version of Record - Feb 8, 2012

What is This? 


\title{
Occupational therapy and sensory integration for children with autism: a feasibility, safety, acceptability and fidelity study
}

\author{
Roseann C. Schaaf
}

Thomas Jefferson University, Pennsylvania, USA

\section{Teal W. Benevides}

Thomas Jefferson University, Pennsylvania, USA

\section{Donna Kelly}

Children's Specialized Hospital, Tom's River, New Jersey, USA

\section{Zoe Mailloux-Maggio}

Pediatric Therapy Network, Torrance, California, USA

\begin{abstract}
Objective: To examine the feasibility, safety, and acceptability of a manualized protocol of occupational therapy using sensory integration principles for children with autism.

Methods: Ten children diagnosed with autism spectrum disorder ages 4-8 years received intensive occupational therapy intervention using sensory integration principles following a manualized protocol. Measures of feasibility, acceptability and safety were collected from parents and interveners, and fidelity was measured using a valid and reliable fidelity instrument.

Results: The intervention is safe and feasible to implement, acceptable to parents and therapist, and therapists were able to implement protocol with adequate fidelity. These data provide support for implementation of a randomized control trial of this intervention and identify specific procedural enhancements to improve study implementation.
\end{abstract}

\section{Keywords}

autism, spectrum disorder, occupational therapy, sensory integration

\section{Introduction}

Sensory impairments are extremely prevalent (80-90\%) in individuals with autism spectrum disorders (ASD) and contribute to the maladaptive behavioral profile of these disorders (Rogers and

Corresponding author:

Roseann C. Schaaf, Thomas Jefferson University, I30 S. 9th Street, Suite 810, Philadelphia, PA 19107, USA.

Email: roseann.schaaf@jefferson.edu 
Table I. Inclusion criteria

I. Diagnosis of autism spectrum disorder based on the ADI-R and the ADOS-G

2. Between the ages of $\mathbf{4 8}$ months and 96 months

3. No physical or medical conditions that affected the child's ability to participate in the active sensory motor activities that are part of the intervention protocol

4. No significant medical or developmental conditions, including Retts disorder, Fragile X, tuberous sclerosis, or blindness/deafness

5. Ability to follow simple verbal directions in English

6. Evidence of sensory dysfunction as measured by the Sensory Profile total test score or the SIPT

7. No current plan to initiate any treatment alternatives during the study period, such as a new restrictive diet program, drug or vitamin therapy

8. Willingness to participate in a 6 week intervention with a frequency of three sessions a week

Ozonoff, 2005). Families report that sensory impairments significantly restrict full participation in daily activities and create social isolation for them and their child (Dickie et al., 2009; Schaaf et al., 2011). Consequently, interventions to address these issues are among the most often requested and used services (Green et al., 2006). Although preliminary data on interventions addressing sensory and motor difficulties in ASD are promising (May-Benson and Koomar, 2010), there is a need for systematic, methodologically rigorous investigations of occupational therapy using sensory integration (OT/SI) to evaluate its safety, acceptability, efficacy, and effectiveness.

Sensory integration is 'the neurological process that organizes sensations from one's body and from the environment and makes it possible to use the body effectively in the environment' (Ayres, 1989, p. 22). The theory and treatment of sensory integration was developed by A. Jean Ayres (Ayres, 1972; 1989), and has mainly been explicated in the field of occupational therapy. Briefly, the theory proposes that if a child is engaged in individually tailored sensory-motor activities, their nervous system is better able to modulate, organize, and integrate sensory information and more likely to use sensory information in adaptive ways (Ayres, 1972). Components of the intervention include a sensory-rich, playful, child-centered approach, providing a just-right challenge (scaffolding) and facilitating progressively more sophisticated adaptive behaviors by engaging the child in individually tailored, developmentally appropriate play interactions (see Schaaf et al., 2009 for greater detail). Treatment is carried out by a therapist trained in the approach and goals are often centered on improving sensory and motor abilities to: a) develop better sensory modulation for attention and behavioral control, and/or b) integrate sensory information as a basis for improved for motor planning (imitation, sequencing, learning novel tasks) as a precursor for greater participation in school, play, social, and daily living activities (Baranek, 2002).

Based on recommendations in the literature for systematically validating and disseminating data on psychosocial interventions in autism (Smith et al., 2007), and given the need for rigorous study of OT/SI, we initiated a feasibility study and follow the recommendations of Zwarenstein et al. (2008) for reporting on pragmatic trials of manualized interventions.

\section{Methods}

\section{Design}

A quasi-experimental pre-test/post-test design was used and ethics approval obtained from the first author's institution. Parental consent and permission to videotape treatment sessions was obtained. 


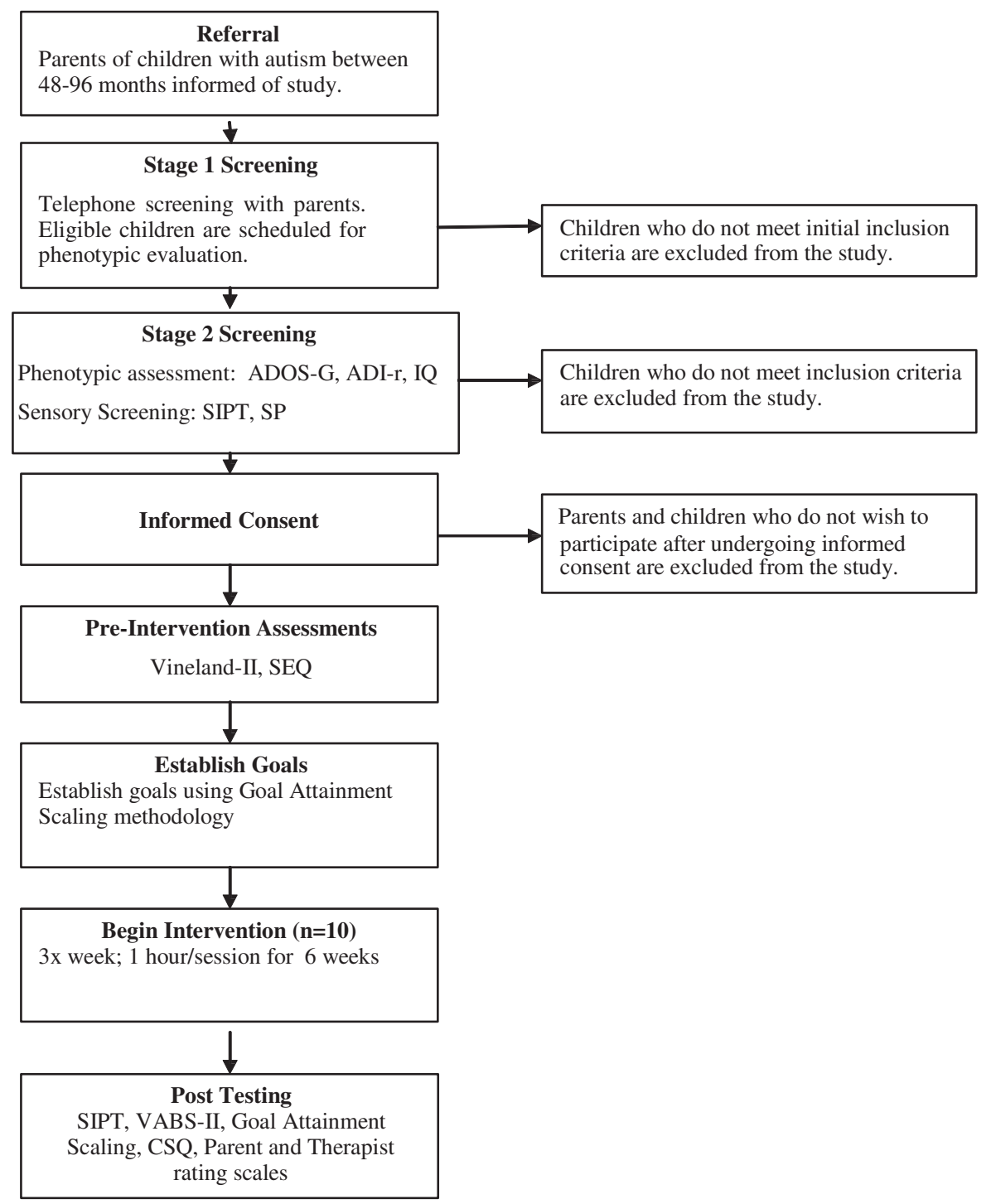

Figure I. Parent and Therapist rating scales - a 12-item study-specific questionnaire to evaluate parent satisfaction. ADOS: Autism Diagnostic Observation Scales (Lord et al., 1999); ADI-R: Autism Diagnostic Interview - Revised (Lord et al., I 994); SIPT: The Sensory Integration and Praxis Tests (Ayres, 1989); SP: Sensory Profile (Dunn, 1999); SEQ: Sensory Experiences Questionnaire (Baranek et al., 2006); Vineland-II: The Vineland Adaptive Behavior Scales; CSQ: Client Satisfaction Questionnaire-8 (Attkisson and Zwick, 1982).

\section{Participants}

Participants were recruited from the clinical population at Children's Specialized Hospital in New Jersey. Twenty-two families inquired about the study, and the first ten children who met inclusion criteria were enrolled. Eight males and two females diagnosed with ASD using the Autism Diagnostic Observation Schedule - Generic (ADOS-G) and Autism Diagnostic Interview - revised 
(ADI-R) met the inclusion criteria (see Table 1). Participants were between 48 and 96 months of age $(62.43 \pm 13.13)$ and IQ scores ranged from 38 to $109(81.00 \pm 27.19)$; Vineland Adaptive Behavior Scales-II Adaptive Behavior Composite Scores ranged from 69 to $94(81.40 \pm 8.85)$.

\section{Procedures}

Autism diagnosis was confirmed by an independent psychologist using the ADI-R (Lord et al., 1994) and the ADOS-G (Lord et al., 1999) and measurement of cognitive level was completed using the Stanford-Binet scale, the Differential Abilities Scale, or the Wechsler Preschool and Primary Scale of Intelligence. Participants were then evaluated to confirm sensory impairment using the Sensory Integration and Praxis Test (SIPT; Ayres, 1989) and the Sensory Profile (Dunn, 1999). Next, an independent evaluator met with the parents to identify and scale goals following standardized goal attainment scaling as described by Kiresuk et al. (1994). Figure 1 shows the participant flow through the study including screening and outcome measures used at each stage.

\section{Occupational therapy intervention}

The intervention was provided three times per week in 1-hour sessions for 6 weeks and followed a manualized protocol based on Ayres (1972) sensory integration principles. The intervention was delivered by two registered, licensed occupational therapists certified in sensory integration, and who were trained on the manual. The therapist followed a guided data driven intervention process as outlined in the intervention manual to design individually tailored treatment activities considering the child's unique strengths and limitations. The intervention is not designed to be a comprehensive treatment for autism but rather a component of a comprehensive program that includes educational, behavioral, and medical approaches.

\section{Fidelity}

Treatment integrity was confirmed using the Ayres Sensory Integration ${ }^{\circledR}$ Fidelity Measure (Parham et al., 2007). The measure has ten items, weighted and rated on a Likert scale of one to five, with five being strong agreement that the therapist used a particular component of the approach. A score of 100 indicates perfect adherence to an intervention and a score of 80 or above is considered acceptable (Parham et al., 2007). This measure has been found to have an inter-rater reliability of .988 for total fidelity score, with individual item inter-rater reliability ranging from .94 to .99 . Validity has been found to be strong as raters were accurately able to distinguish OT/SI sessions from other intervention approaches with $92 \%$ accuracy. All treatment sessions were videotaped and random selections of $20 \%$ were evaluated by independent evaluators trained to use the instrument.

\section{Results}

Descriptive statistics were used to summarize parent and therapist rating scales and therapist fidelity and to document adverse events. Frequency counts of existing and missing data were used as a measure of the feasibility of administering the protocol.

\section{Feasibility}

Nine out of the 10 children completed the study, reflecting a retention rate of $90 \%$. One parent/ child decided not to participate. 
We also evaluated missing data as a measure of feasibility of enacting the study protocol in this setting. We compared the total number of potential data sources with the actual number. Seventytwo percent of the pre-test data and $71 \%$ of the post test data were obtained.

In regard to parent perceptions of the feasibility of participating in the protocol, of the six parents who completed the post test feasibility rating, all indicated that it was easy or very easy to schedule treatment and to get to the facility, and that sessions were delivered on time.

Therapists carrying out the protocol agreed or strongly agreed that training on the study protocol was satisfactory for conducting the treatment sessions and that the time provided for intervention sessions was adequate to meet the child's goals, but that the time for documentation of the child's intervention was inadequate $75 \%$ of the time.

\section{Safety}

Frequency of adverse events affecting participants and study therapists were used to document safety and parent-reported perception of safety and acceptability of the intervention. There were no reports of injury among the children or therapists during the study period, indicating that the protocol was safe to deliver. Of the six parents who completed the parent rating of treatment questionnaire at posttest, all rated the environment as safe and rated the therapist as being aware of their child's safety.

\section{Satisfaction}

All seven of the parents who completed client satisfaction questionnaires indicated that they were 'very satisfied' with treatment and that the treatment received through the study was 'good' or 'excellent'. One hundred percent of these parents agreed that the intervention helped them deal with challenges faced in daily life.

\section{Fidelity ratings}

A random sampling of $20 \%$ of the videotapes of the treatment sessions were rated for therapist's fidelity as recommended by Parham et al. (2007), yielding a total of 33 fidelity ratings. Scores ranged from 63 to 97 with a mean of 82, showing that therapists were able to maintain acceptable fidelity to the treatment protocol. Lower scores were obtained early in the study and, thus, the data driven intervention process from the manual was further explicated to guide the therapist reasoning and remedy this gap.

\section{Discussion}

We follow the recommendations of Smith et al. (2007) in the design of our clinical trial and begin with this report on the safety, feasibility, acceptance and therapist's fidelity to the manualized intervention. Although there are several studies of occupational therapy using sensory integration (see May-Benson and Koomar, 2010, for a review), none specifically address the safety and feasibility of implementation, few measure treatment integrity, and only a handful evaluate its application to children with autism. Therefore, the acceptability of this intervention by other professionals is often questioned and rigorous study is sorely needed. Our findings take an important, initial step toward rigorous study of OT/SI, showing that our manualized protocol is safe, feasible to attend and administer, parents are satisfied, and that therapists are able to maintain adequate adherence to treatment (fidelity). 
Intervention studies on clinical populations are complex because of the multifaceted issues associated with the participants and their families. This complexity is further exacerbated by the challenges inherent in studying young children with autism, who make up a very heterogeneous population (Spence and Thurm, 2010). We found that procedural rigor with timely follow-up was essential for compliance with data collection. We identified the need for specific procedural checklists that monitor data collection, chart reviews to assure that these procedures are followed, and administrative assistance to improve data collection and documentation. We also found that explanation of the data driven intervention process (added after two participants were enrolled) was extremely valuable for the interveners, allowing them to implement the intervention with high fidelity. The data driven intervention process outlines the proposed hypotheses for the child's participation limitations (based on assessment findings) and guides the therapist in the expeditious design, conduct, and documentation of intervention while maintaining fidelity to the principles and practices outlined in the manual. These procedural enhancements were designed to decrease the number of missing data in future studies and improve therapists' adherence to the protocol, and they will be useful in future studies.

An important consideration for rigorous clinical trials is assurance that outcome measures are sensitive to detecting change in outcomes that are both close to the intervention's intent (proximal outcomes) and meaningful for the child and family in their daily lives (distal outcomes) (Lord et al., 2005). We evaluated changes in sensory processing and praxis (proximal outcome) while simultaneously measuring changes in adaptive behavior, participation and family-identified functional goals (distal outcomes), as outlined in Figure 1. In particular, we found that goal attainment scaling, a standardized method of documenting progress toward individual, participation-oriented goals, was useful for capturing the changes in function/participation that may be linked to changes in sensory processing as a result of the intervention. Of the six complete goal attainment scales data sets, four of the children achieved a $\mathrm{T}$ score of greater than 50 (range 68-78), indicating above expected achievement in their individual goals, and these results were corroborated by parent and therapist reports. Thus, goal attainment scaling does show promise as an outcome measure for the larger randomized trial. The SIPT and the Vineland-II also show promise as outcome measures for OT/SI as data were in the expected directions; however, no conclusions can be reached at this time owing to the limited data set.

\section{Conclusion}

OT/SI is safe, acceptable and feasible to administer in a short, intensive manner for children with autism and these data support the implementation of a larger randomized controlled trial. Specific procedural enhancements were identified to improve data collection, documentation, and therapists' fidelity and will be used in the randomized controlled trial phase of the study.

\section{References}

Attkisson CC and Zwick RJ (1982) The Client Satisfaction Questionnaire: Psychometric properties and correlations with service utilization and psychotherapy outcome. Evaluation and Program Planning 5(3): 233-237.

Ayres AJ (1972) Sensory integration and learning disorders. Los Angeles: Western Psychological Services. Ayres AJ (1989) The sensory integration and praxis tests. Los Angeles: Western Psychological Services.

Baranek GT (2002) Efficacy of sensory and motor interventions for children with autism. Journal of Autism and Developmental Disorders 32(5): 397-422. 
Baranek, G. T., David, F. J., Poe, M. D., Stone, W. L., \& Watson, L. R. (2006). Sensory experiences questionnaire: Discriminating sensory features in young children with autism, developmental delays, and typical development. Journal of Child Psychology and Psychiatry and Allied Disciplines, 47(6), 591-601.

Dickie V, Baranek G, Schultz B, Watson L and McComish C (2009) Parent reports of sensory experiences of preschool children with and without autism: A qualitative study. American Journal of Occupational Therapy 63(2): 172-181.

Dunn W (1999) The Sensory Profile User's Manual. Ontario Canada: Harcourt.

Elliot, C.D. (2007). Differential Ability Scales - second Edition (DAS-II). San Antonio, TX: Psychological

Corporation.

Green V, Pituch K, Itchon J, Choi A, O'Reilly M and Sigafoos J (2006) Internet survey of treatments used by parents of children with autism. Research in Developmental Disabilities 27(1): 70-84.

Kiresuk TJ, Smith A and Cardillo JE (1994) Goal attainment scaling: Applications, Theory and Measurement. Hillsdale, NJ: Erlbaum.

Lord C, Rutter M and Le Couteur A (1994) Autism diagnostic interview-revised: A revised version of a diagnostic interview for caregivers of individuals with possible pervasive developmental disorders. Journal of Autism and Developmental Disorders 24(5): 659-685.

Lord C, Rutter M, DiLavore P and Risi S (1999) The Autism Diagnostic Observation Schedule - Generic. Los Angeles: Western Psychological Services.

Lord C, Wagner A, Rogers S, Szatmari P, Aman M, Charman T, et al. (2005) Challenges in evaluating psychosocial interventions for autistic spectrum disorders. Journal of Autism and Developmental Disorders 35(6): 695-708.

May-Benson T and Koomar J (2010) Systematic review of the research evidence examining the effectiveness of interventions using a sensory integrative approach for children. American Journal of Occupational Therapy 64(3): 403-414.

Parham LD, Cohn ES, Spitzer S, Koomar JA, Miller LJ, Burke JP, et al. (2007) Fidelity in sensory integration intervention research. American Journal of Occupational Therapy 61: 216-227.

Rogers SJ and Ozonoff S (2005) Annotation: What do we know about sensory dysfunction in autism? A critical review of the empirical evidence. Journal of Child Psychology and Psychiatry 46(12): 1255-1268.

Roid, G. H. (2003). Stanford-Binet Intelligence Scales, Fifth Edition. Itasca, IL: Riverside Publishing.

Schaaf RC, Schoen SA, Smith Roley S, Lane SJ, Koomar JA and May-Benson TA (2009) A frame of reference for sensory integration. In: Kramer P and Hinojosa J (eds) Frames of Reference for Pediatric Occupational Therapy, $3^{\text {rd }}$ edn. Philadelphia: Lippincott Williams and Wilkins, pp. 99-186.

Schaaf RC, Benevides T, Johnson S, Madrid G and Toth-Cohen S (2011) The everyday routines of families of children with autism: Examining the impact of sensory processing difficulties on the family. Autism: The International Journal of Research and Practice 15(3): 373-389.

Smith T, Scahill L, Dawson G, Guthrie D, Lord C, Odom S, et al. (2007) Designing research studies on psychosocial interventions in autism. Journal of Autism and Developmental Disorders 37(2): 354-366.

Spence SJ and Thurm A (2010) Testing autism intervention: Trials and tribulations. The Lancet 375(9732): 2124-2125.

Wechsler, D. (1989) Wechsler Preschool and Primary Scale of Intelligence. San Antonio, TX: Psychological Corp.

Zwarenstein M, Treweek S, Gagnier JJ, Altman DG, Tunis S, Haynes B, et al. (2008) Improving the reporting of pragmatic trials: An extension of the CONSORT statement. British Medical Journal 337: 1-8. 